\title{
Analytical XTEM Study of Ir/InAIAs Interfacial Reaction for InP-based High Electron Mobility Transistors (HEMTs) Gate Technology
}

\author{
Liang Wang, ${ }^{1,2}$ Weifeng Zhao, ${ }^{1,3}$ and Ilesanmi Adesida ${ }^{1,2,3}$
}

1. Micro and Nanotechnology Lab, 2. Dept Materials Science and Engineering, and 3. Dept Electrical and Computer Engineering, University of Illinois at Urbana-Champaign, Urbana, IL 61801

Schottky contact property is one of the essential concerns for the application of InP-based HEMTs where high Schottky barrier height $\left(\varphi_{\mathrm{B}}\right)$ is desirable for the reduction of gate leakage current and tailoring of threshold voltage. Thermal annealing is used to activate the metallization and to obtain high $\varphi_{\mathrm{B}}$. For example, the conventional Pt gate has a $\varphi_{\mathrm{B}}$ of $700 \mathrm{meV}$ when deposited on InAlAs. While upon annealing at $200{ }^{\circ} \mathrm{C}, \varphi_{\mathrm{B}}$ increases to $800 \mathrm{meV}$ [1]. Further annealing causes performance degradation. The low annealing temperature $\left(\mathrm{T}_{\mathrm{a}}\right)$ of $\mathrm{Pt}$ gate makes the reliable performance of devices a big concern. XTEM study demonstrated that prolonged thermal storage of Pt contacts resulted in extensive in-diffusion of Pt into the semiconductor [2] and a failure of the device could thus occur. Alternatively, an Ir-based metallization scheme with comparable $\varphi_{\mathrm{B}}$ to Pt but higher $\mathrm{T}_{\mathrm{a}}$ has been developed by our group [1]. $\varphi_{B}$ as a function of $T_{a}$ for the Ir-based gate to InAlAs/InGaAs HEMT layers is plotted in Fig. 1. Below the optimal condition $\left(\mathrm{T}_{\mathrm{a}}=400{ }^{\circ} \mathrm{C}\right), \varphi_{\mathrm{B}}$ increases monotonically. Beyond this temperature, an over annealing occurs and $\varphi_{\mathrm{B}}$ drops gradually. In present study, a TEM investigation is carried out on samples with different $\mathrm{T}_{\mathrm{a}}$, namely, $325{ }^{\circ} \mathrm{C}, 425{ }^{\circ} \mathrm{C}$ and $475{ }^{\circ} \mathrm{C}$ which correspond to under-annealing, optimal annealing and over-annealing, respectively, to elucidate the Schottky barrier formation mechanism and the over-annealing mechanism.

The epilayer structure and $\varphi_{\mathrm{B}}$ measurement procedure could be found elsewhere [2]. The e-beam deposited gate contacts consisted of $\operatorname{Ir}(10 \mathrm{~nm}) / \mathrm{Ti}(20 \mathrm{~nm}) / \mathrm{Pt}(10 \mathrm{~nm}) / \mathrm{Au}(160 \mathrm{~nm})$ which was then annealed for $30 \mathrm{~s}$ under $\mathrm{N}_{2}$ ambient. XTEM samples were prepared by sample gluing, grinding and ion milling. TEM works were done on a JEOL 2010F TEM for microstructure and microchemistry characterization. The layered structure of the as deposited contacts is clearly revealed by Z-contrast image in Fig. 2a. The Ir layer has polycrystalline morphology from the HRTEM image in Fig. 2b. No interfacial reaction between Ir and InAlAs could be resolved and $\mathrm{Ir}$ is in intimate contact with InAlAs. When annealed at $325{ }^{\circ} \mathrm{C}$ (Fig. 3), Ir diffuses into InAlAs and forms an interfacial amorphous layer (a-layer) with a depth of $\sim 1.2 \mathrm{~nm}$. The formation of a-layer has been observed in several systems such as metal/Si [3] and Pt/InAlAs [2]. We believe that the formation of the a-layer and its composition is essential for Schottky barrier formation. The a-layer is too thin to confidently obtain the composition by EDS though. Further annealing to the optimal condition yields an even thicker a-layer $(2.5 \mathrm{~nm})$ as shown in Fig. 4a. EDS composition profiles across the metallization is drawn in Fig. 4b. The Ir layer is thick enough to block Ti in-diffusion; out-diffusion of As and In from the epilayer is manifested suggesting the a-layer consists these two elements. Annealing at 475 ${ }^{\circ} \mathrm{C}$ creates significant different interfacial structure and that the a-layer starts to crystallize (Fig. 5). This crystallized layer has a thickness of $\sim 4 \mathrm{~nm}$ which means a significant portion (50\%) of the InAlAs layer is consumed. EDS analysis indicates these crystals have an average composition of $\sim 34$ $\%$ Ir (at. \% here and after), $48 \%$ As and 18\% In. An investigation of Ir-Al-As and Ir-In-As ternary phase diagrams indicates that $\operatorname{IrAs}_{2}$, IrAl or $\operatorname{IrIn}_{2}$ could be the crystal phases. The results suggest that the a-layer is the $\varphi_{\mathrm{B}}$ formation mechanism in Ir/InAlAs. The formation of stable crystalline phases or the consumption of the InAlAs layer may degrade contact performance. 


\section{References}

[1] S. Kim et al., Electron. Lett., 41 (2005) 665.

[2] S. Kim et al., Appl. Phys. Lett., 87 (2005) 232102.

[3] L.J. Chen et al, Mater. Sci. Eng. R29 (2000) 115.

[4] This work was supported by NSF ANI (01-21662 ITR) and ONR (N00014-01-1000). TEM works were carried out at the Center for Microanalysis of Materials at the University of Illinois (DOEFG02-91-Er45439). Assistance of J.G. Wen and C.H. Lei on the TEM is acknowledged.

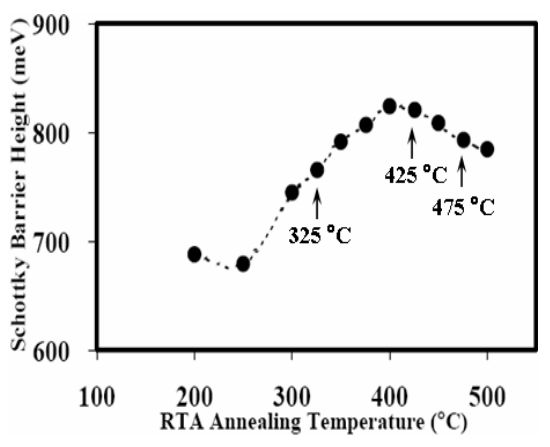

FIG. 1. $\varphi_{\mathrm{B}}$ as a function of $\mathrm{T}_{\mathrm{a}}$ for Ir gate metallization on InAlAs/InGaAs

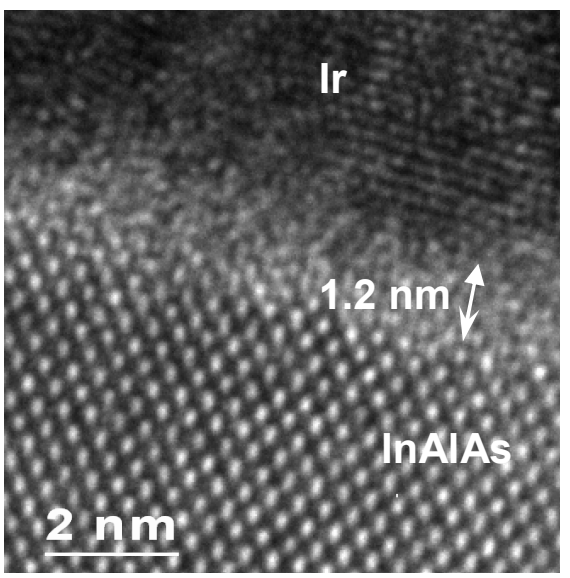

FIG. 3. HRTEM image of after annealed at $325^{\circ} \mathrm{C}$

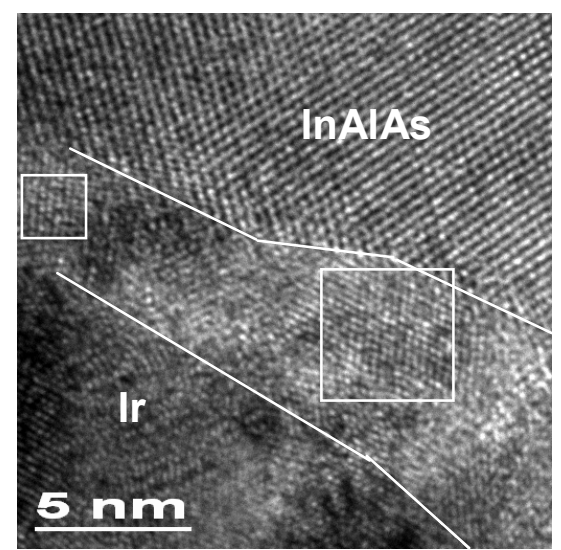

FIG. 5. HRTEM image of after annealed at $475{ }^{\circ} \mathrm{C}$
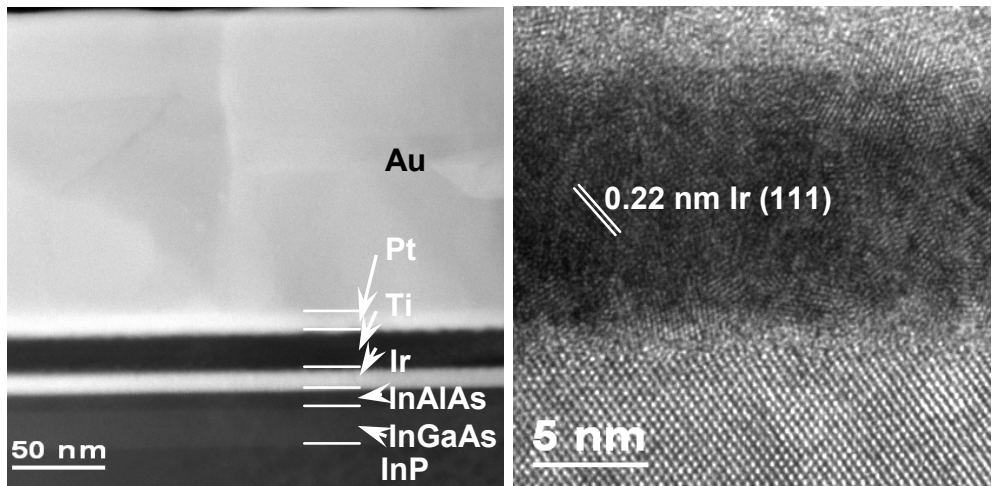

FIG. 2. As deposited structure. (a) Z-contrast image, (b) HRTEM image of the Ir/InAlAs layers.
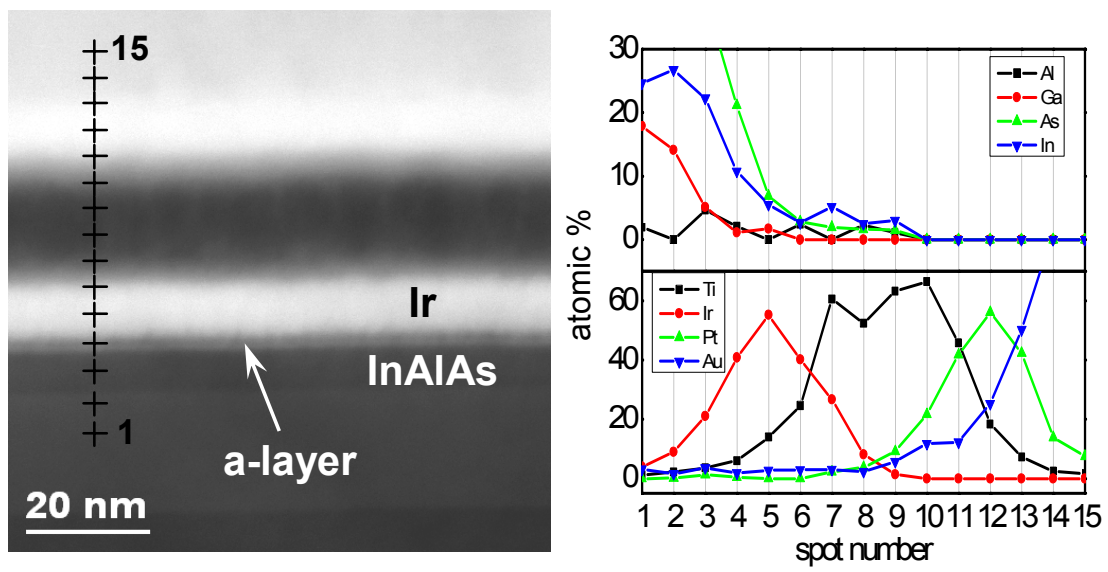

FIG. 4. (a) Z-contrast image after annealed at $425{ }^{\circ} \mathrm{C}$. (b) EDS composition profiles. 